\title{
Dynamics of a chain of magnetic particles connected with elastic linkers
}

\author{
Andrejs Cebers \\ Institute of Physics, Salaspils-1, LV-2169, Latvia \\ E-mail: aceb@sal.lv
}

Received 17 September 2002

Published 7 April 2003

Online at stacks.iop.org/JPhysCM/15/S1335

\begin{abstract}
The model of an elastic magnetic chain is considered. By numerical simulation of the dynamics of rod shapes acted on by magnetic and elastic forces in viscous fluid, it is shown that the characteristic ' $U$ '-like metastable shapes (hairpins) can be formed. The characteristic ' $S$ '-like long-living shapes are formed at intermediate stages, depending on the initial perturbation of the rod, and finally relax to the global energy minima corresponding to the straight rod. Further extensions of the model will include long-range magnetic interactions between distant parts of a rod.
\end{abstract}

\section{Introduction}

Chains of dipolar particles have been well studied in relation to the phase separation in dipolar systems (for a review, see Bossis 2002). Approaches based on the statistical physics of colloidal systems have shown that screening of dipolar interactions due to the formation of chains could be responsible for the absence of phase coexistence in systems of dipolar hard spheres (see Sears 1996, van Roij 1996, Levin 1999, Teixeira et al 2000) observed in numerical experiments (Weis and Levesque 1993). Recently. a new mechanism of phase separation of magnetic colloids due to branching of chains of dipolar particles has been considered (Tlusty and Safran 2000). The chains of dipolar particles also have interesting dynamic properties. It was shown that a chain of magnetic particles acted on by a rotating field with large enough frequency forms, before breaking, characteristic ' $S$ '-like shapes (Melle et al 2002). A theoretical model of the formation of the ' $S$ ' shapes by viscous magnetic threads has been proposed in Cebers (2002). There, by numerical simulation of the nonlinear partial differential equation (PDE) describing the dynamics of the thread in the rotating field, it was shown that the ' $S$ ' shape arises by the propagation of jumps of the thread's centreline tangent angle. The dependence of the critical frequency of the jump formation on the length of the droplet $L$ obtained allows one to derive the scaling law $L \sim f^{-1 / 2}$ for the dependence of the chain length on the frequency $f$ of the rotating field (Melle et al 2002). Such a law arises quite naturally in the framework of the 
model proposed, assuming that the upper bound for the chain length growth due to the dipolar interactions is limited by the process of ' $S$ '-shape formation leading to the break-up of the chain (Sandre et al 1999). Quite interesting and new objects can be obtained by connecting the magnetic particles by elastic linkers (Furst et al 1998, Furst and Gast 1999). At present there are several interesting ideas for the application of chains of magnetic particles as diagnostic tools for establishing the presence of specific antibodies and for the creation of media for the fractionation of DNA by electrophoresis etc (Mayer et al 1997, Doyle et al 2002).

Here, a model of the magnetoelastic behaviour of the magnetizable elastic rod acted on by elastic and viscous forces and elastic and magnetic torques is proposed. In the framework of the model, the response of the rod to an abruptly applied perpendicular field is considered and it is shown that the initially straight rod can establish ' $U$ '-like metastable shapes (hairpins) and long-living ' $\mathrm{S}$ '-like configurations, depending on the ratio of the elastic and magnetic forces, finally relaxing to the straight shape corresponding to global energy minima.

\section{Model}

As the model for the chain of magnetizable particles connected by elastic linkers, the Kirchhoff model of a nonstretchable elastic rod is chosen. The magnetic energy of the rod and thus the magnetic torque on the rod are accounted for in the spirit of the slender-body approach, locally approximating the magnetic rod as a long straight cylinder. For the dynamics of the magnetic rod in viscous media, the simplest approach of Rouse dynamics is taken. A nonlinear PDE is formulated for the centreline's tangent angle.

According to the Kirchhoff model of an elastic rod, extended by including the magnetic term, the energy has the form (Landau and Lifshitz 1965)

$$
E=\frac{1}{2} C \int \frac{1}{R^{2}} \mathrm{~d} l-\frac{2 \pi^{2} a^{2} \chi^{2} H_{0}^{2}}{\mu+1} \int(\vec{h} \cdot \vec{n})^{2} \mathrm{~d} l .
$$

Here $R$ is the radius of curvature of the centreline, while $C$ is that of the cylinder of radius $a$, and its Young modulus $Y$ is expressed as follows: $C=\frac{\pi}{4} a^{4} Y ; \mu=1+4 \pi \chi, \chi$ is the magnetic susceptibility of the rod, $H_{0} \vec{h}$ is the external magnetic field strength in the direction of the unit vector $\vec{h}, \vec{n}$ is the normal to the centreline of the rod. We further consider the case where a constant magnetic field is applied along the $x$-axis. The tangent to the centreline $\vec{t}$ is given by its components: $\vec{t}=(\cos (\theta), \sin (\theta))$. The local nonstretchability of the rod is accounted for by introducing the local tension of the rod as a Lagrange multiplier, $\Lambda$. The total energy functional determining the elastic forces on the rod has the form

$$
E=\frac{1}{2} C \int \frac{1}{R^{2}} \mathrm{~d} l-\frac{2 \pi^{2} a^{2} \chi^{2} H_{0}^{2}}{\mu+1} \int(\vec{h} \cdot \vec{n})^{2} \mathrm{~d} l-\int \Lambda \mathrm{d} l .
$$

Considering the variation of (1) with respect to $\vec{r}^{\prime}=\vec{r}+\vec{\xi}$, the following expression for the first variation of the energy functional is obtained:

$$
\delta E=[M \delta \varphi]+\left[F_{t} \xi_{t}\right]+\left[F_{n} \xi_{n}\right]-\int K_{n} \xi_{n} \mathrm{~d} l-\int K_{t} \xi_{t} \mathrm{~d} l .
$$

Here [ ] denotes the values at the ends of the rod, $\xi_{n}$ and $\xi_{t}$ are components of the Lagrange displacement in the directions of the normal and tangent to the centreline respectively, but $\delta \varphi=\frac{\partial \xi_{n}}{\partial l}-\frac{\xi_{t}}{R}$ is the angle of tangent rotation at the Lagrange displacement positions $\vec{\xi}$. The tangent and normal vectors are connected according to the Frenet equation $\frac{\mathrm{d} \vec{t}}{\mathrm{~d} l}=-\frac{1}{R} \vec{n}$, where $l$ is arc length of the rod's centreline and $R$ is the radius of curvature of the centreline. The binormal $\vec{b}$ to the centreline is defined by $\vec{b}=[\vec{t} \times \vec{n}]$. According to the relation (2), the 
following expressions for the components of the body force $\vec{K}$, stresses $\vec{F}$ and momentum stresses $\vec{M}=M \vec{b}$ are valid:

$F_{n}=C\left(\frac{1}{R}\right)_{l}+\frac{2 \pi^{2} a^{2} \chi^{2} H_{0}^{2}}{\mu+1} \sin (2 \theta)$
$F_{t}=-\left(\frac{C}{2 R^{2}}+\Lambda\right)$
$K_{n}=\frac{\mathrm{d} F_{n}}{\mathrm{~d} l}-\frac{F_{t}}{R}=C\left(\left(\frac{1}{R}\right)_{l l}+\frac{1}{2} \frac{1}{R^{3}}\right)+\Lambda \frac{1}{R}+\frac{2 \pi^{2} a^{2} \chi^{2} H_{0}^{2}}{\mu+1} \frac{\mathrm{d}(\sin (2 \theta))}{\mathrm{d} l}$
$K_{t}=\frac{\mathrm{d} F_{t}}{\mathrm{~d} l}+\frac{F_{n}}{R}=-\Lambda_{l}+\frac{1}{R} \frac{2 \pi^{2} a^{2} \chi^{2} H_{0}^{2}}{\mu+1} \sin (2 \theta)$
$M=-\frac{C}{R}$.

It can be easily obtained that

$$
\vec{K}=\frac{\mathrm{d} \vec{F}}{\mathrm{~d} l}=\frac{\mathrm{d}}{\mathrm{d} l}\left(F_{n} \vec{n}+F_{t} \vec{t}\right)
$$

In the simplest case of Rouse dynamics, when the hydrodynamic interaction between the particles in the chain is neglected, we have

$$
\zeta \vec{v}=\vec{K}
$$

Here $\zeta$ is friction coefficient of the chain per unit length. As we can see from the expressions (3)-(7), in the case of a magnetic rod, additional normal stresses $F_{n}^{\prime}=$ $\frac{2 \pi^{2} a^{2} \chi^{2} H_{0}^{2}}{\mu+1} \sin (2 \theta)$ arise, which are necessary to balance the magnetic torque $\vec{T}_{0}$. In this case the condition of torque balance for rotational equilibrium of the rod reads

$$
\frac{\partial \vec{M}}{\partial l}+[\vec{t} \times \vec{F}]+\vec{T}_{0}=0
$$

or $F_{n}^{\prime}=-T_{0}$. Here the magnetic torque in the magnetic field along the $x$-axis is calculated according to the formula

$$
T_{0}=-\frac{2 \pi \chi^{2} H_{0}^{2} \sin (2 \theta) \pi a^{2}}{\mu+1} .
$$

The equation for the tangent angle reads

$$
\frac{\mathrm{d} \theta}{\mathrm{d} t}=\frac{\partial v_{n}}{\partial l}-\frac{v_{t}}{R}
$$

where, due to nonstretchability, $\frac{\mathrm{d} l}{\mathrm{~d} t}=0$. The condition of nonstretchability has the form

$$
\frac{\partial v_{t}}{\partial l}+v_{n} \frac{1}{R}=0
$$

Expressing the velocity components according to relation (9) and taking into account (5) and (6), we obtain the equation for the tension $\Lambda$ in the rod:

$-\Lambda_{l l}+\frac{1}{R^{2}} \Lambda+\frac{C}{R}\left(\left(\frac{1}{R}\right)_{l l}+\frac{1}{2 R^{3}}\right)+\frac{1}{R} \frac{\partial F_{n}^{\prime}}{\partial l}+\frac{\partial}{\partial l}\left(\frac{1}{R} \frac{2 \pi^{2} a^{2} \chi^{2} H_{0}^{2}}{\mu+1} \sin (2 \theta)\right)=0$.

The equation for the tangent angle (12), on taking into account the nonstretchability, takes the form

$$
\zeta \frac{\partial \theta}{\partial t}=C\left[\left(\frac{1}{R}\right)_{l l l}+\frac{1}{2} \frac{\partial}{\partial l}\left(\frac{1}{R^{3}}\right)\right]+\frac{\partial}{\partial l}\left(\frac{\Lambda}{R}\right)+\frac{\partial^{2} F_{n}^{\prime}}{\partial l^{2}}-\frac{1}{R^{2}} \frac{2 \pi^{2} a^{2} \chi^{2} H_{0}^{2}}{\mu+1} \sin (2 \theta)+\frac{\Lambda_{l}}{R} .
$$


Since $\frac{1}{R}=-\frac{\partial \theta}{\partial l}$, equation (15) can be written in the form

$$
\begin{aligned}
\zeta \frac{\partial \theta}{\partial t}=C\left(-\theta_{l l l l}\right. & \left.-\frac{1}{2} \frac{\partial}{\partial l}\left(\frac{\partial \theta}{\partial l}\right)^{3}\right)-\frac{\partial}{\partial l}\left(\frac{\partial \theta}{\partial l} \Lambda\right)-\Lambda_{l} \frac{\partial \theta}{\partial l} \\
+ & \frac{2 \pi^{2} a^{2} \chi^{2} H_{0}^{2}}{\mu+1} \frac{\partial^{2} \sin (2 \theta)}{\partial l^{2}}-\left(\frac{\partial \theta}{\partial l}\right)^{2} \frac{2 \pi^{2} a^{2} \chi^{2} H_{0}^{2}}{\mu+1} \sin (2 \theta) .
\end{aligned}
$$

Introducing the characteristic elastic timescale $\tau=\frac{C}{L^{4} \zeta}$, where $2 L$ is the length of the rod, the equation for the tangent angle has the following dimensionless form:

$\theta_{t}=-\left(\theta_{l l l l}+\frac{1}{2}\left(\theta_{l}^{3}\right)_{l}\right)-\left(\theta_{l} \Lambda\right)_{l}-\Lambda_{l} \theta_{l}+C m(\sin (2 \theta))_{l l}-C m\left(\theta_{l}\right)^{2} \sin (2 \theta)$.

Correspondingly, the equation for the tension of the rod put in dimensionless form is

$$
\theta_{l}^{2} \Lambda-\Lambda_{l l}=-\theta_{l}\left(\theta_{l l l}+\frac{1}{2} \theta_{l}^{3}\right)+C m \theta_{l}^{2} 2 \cos (2 \theta)+C m\left(\theta_{l} \sin (2 \theta)\right)_{l} .
$$

Here $C m=\frac{2 \pi \chi^{2} H_{0}^{2} \pi a^{2} L^{2}}{(\mu+1) C}$ is the magnetoelastic number characterizing the ratio of the magnetic and elastic forces. The coupled set of equations (17) and (18) describing the dynamics of the rod acted upon by the magnetic and elastic forces in the viscous fluid is solved numerically. The numerical scheme used is described in the appendix. The boundary conditions which should be added to the set of equations to express the conditions of absence of stresses and torque at the free ends of the rod are

$$
\begin{aligned}
& -\theta_{l l}+C m \sin (2 \theta)=0 \\
& \theta_{l}=0
\end{aligned}
$$

and

$$
\Lambda( \pm 1)=0
$$

\section{Numerical simulation results and discussion}

A question which arises regarding elastic magnetic chains is: could some other shapes, different from the straight trivial one, exist under the influence of the magnetic field? As a possible candidate, we consider hairpins, which are found in different systems among which the nematic polymers are especially interesting (Warner et al 1985, Gunn and Warner 1987). Hairpin formation by elastic rods, as obtained by numerical simulation according to equations (17) and (18), is shown in figure 1. As the initial state, the slightly bent shape described by the relation

$$
\theta=\arccos \left(0.1 \sin \left(\frac{\pi}{2} l\right)\right)
$$

was chosen. The magnetoelastic number $C m=26$. The number of uniform mesh points here and subsequently is 300 . The simulation of the dynamics shows that, after a transitory period, a steady ' $U$ '-like shape is formed. To study the stability of the hairpin, an asymmetry in the initial state described by the relation

$$
\theta=\arccos \left(0.1 \sin \left(\frac{\pi}{2}\left(l-l_{0}\right)\right)\right)
$$

was introduced. As the results in figure 2 show, a stable hairpin configuration exists also with nonequal lengths of the branches. In this respect, the situation is different from what takes place for a nematic polymer with the dipolar moments along the backbone of the polymer molecule (Gunn and Warner 1987). In the latter case, one of the branches with dipolar moments opposite 

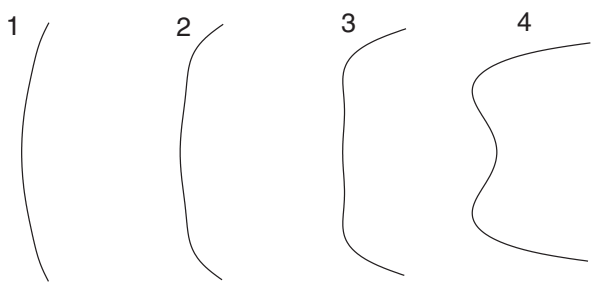

5

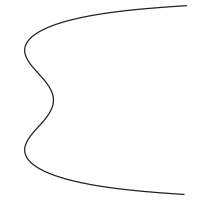

7

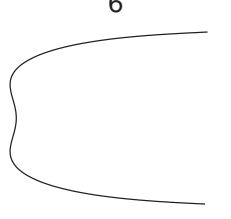

8

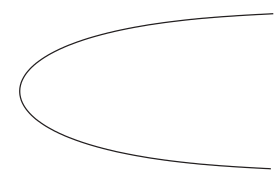

Figure 1. Formation of the 'U'-like metastable shape from symmetric initial conditions Dimensionless time: 0.000069 (1); 0.000347 (2); 0.000694 (3); 0.001736 (4); 0.003472 (5); 0.005208 (6); 0.006944 (7); 0.133333 (8). $\mathrm{Cm}=26$.

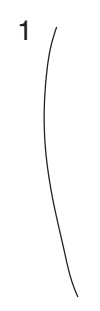

4
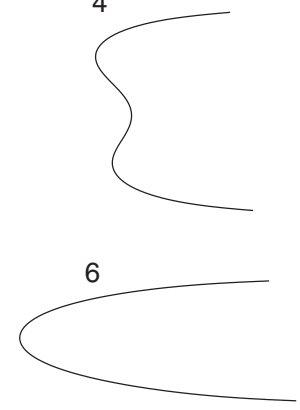

2
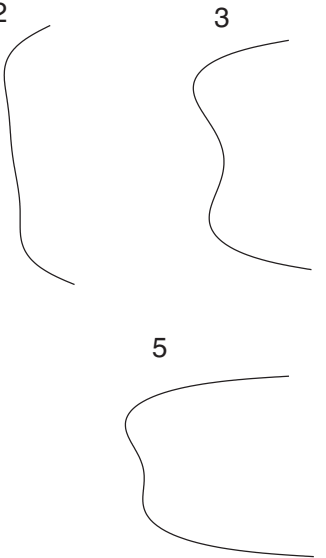

7

Figure 2. Formation of the 'U'-like metastable shape from nonsymmetric initial conditions. Dimensionless time: 0.000069 (1); 0.000694 (2); $0.001736(3) ; 0.003472$ (4); 0.005208 (5); $0.010417(6) ; 0.133333$ (7). $C m=26, l_{0}=1 / 3$.

to the direction of the electric field is very energetically disadvantageous, which is the reason for the giant electric polarizability of such systems (Gunn and Warner 1987). In the case 


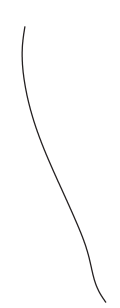

5

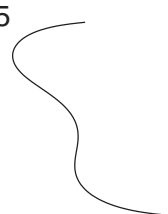

7

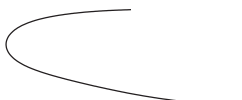

4
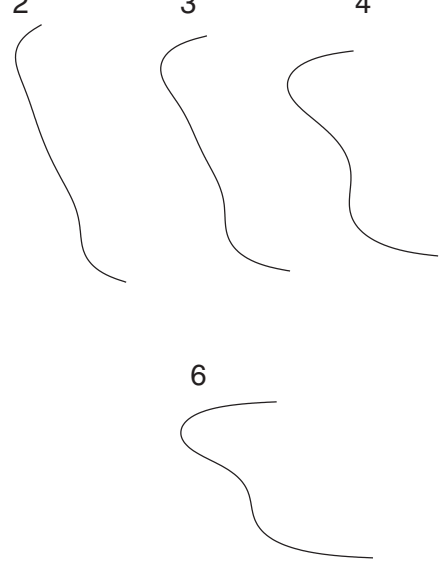

Figure 3. Formation of the highly nonsymmetric ' $U$ '-like metastable shape from nonsymmetric initial conditions. Dimensionless time: 0.000069 (1); 0.000347 (2); 0.000694 (3); 0.001389 (4); 0.001736 (5); 0.003472 (6); 0.006944 (7); 0.133333 (8). $C m=50, l_{0}=5 / 6$.
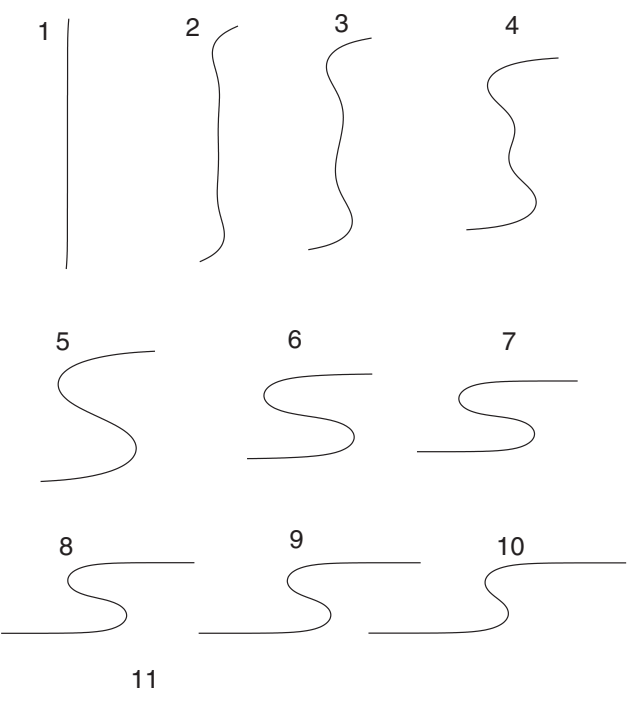

Figure 4. The long-living ' $S$ '-like shape and its relaxation to the straight configuration from an initially unsymmetrically bent shape. Dimensionless time: 0.000069 (1); 0.000347 (2); 0.000694 (3); 0.001736 (4); 0.003472 (5); 0.006944 (6); 0.133333 (7); 0.173611 (8); 0.187500 (9); 0.194444 (10); 0.266667 (11). $C m=50, l_{0}=5 / 6$.

of a polarizable magnetic rod, there is no energy difference between the branches along the magnetic field direction, which makes possible the existence of stable configurations with nonequal lengths of the branches of the hairpins. As shown in figure 3, such configurations 
still exist, as obtained by the numerical simulation starting from the initial configuration given by

$$
\theta=\arccos \left(0.3 \sin \left(\frac{\pi}{2}\left(l-l_{0}\right)\right)\right)
$$

at $l_{0}=5 / 6$ and $C m=50$. Another configuration of interest looks similar to the ' $\mathrm{S}$ '-like shapes. The numerical simulation starting from the initial condition with

$$
\theta=\frac{\pi}{2}-0.01 l^{2}
$$

and $C m=60$ shows that such configurations can arise as long-living transient states, relaxing to the global energy minima corresponding to the straight rod during a long transition period (figure 4). Thus the results of the numerical simulation of the dynamics of the elastic magnetic rods show that such rods can have different metastable states of equilibrium, depending on the initial configurations. Between these states, hairpin configurations with nonequal lengths of the branches are possible. The results of the present numerical simulations show that ' $S$ '-like shapes exist as long-living metastable states. For long-term stability of such configurations, some other physical mechanisms connected for example with the linking of magnetic particles should be involved. Further extension of the model will include long-range magnetic interactions between distant parts of the rod and will also consider the complex dynamics of elastic magnetic rods arising under the influence of rotating magnetic fields.

\section{Appendix}

Equation (17) is solved using an implicit scheme approximating derivatives by finite differences. The nonlinear equation for $\theta^{n+1}$ in the following time step is $(i=2, \ldots, N-2$; uniform mesh points are located at $l_{i}=\mathrm{i} H$; here $H=2 / N$ )

$$
\begin{aligned}
\theta_{i}^{n+1}+\frac{\Delta t}{h^{4}}\left[\theta_{i+2}^{n+1}\right. & \left.+\theta_{i-2}^{n+1}-4 \theta_{i+1}^{n+1}-4 \theta_{i-1}^{n+1}+6 \theta_{i}^{n+1}\right]+\frac{\Delta t}{2 h^{4}}\left[\left(\theta_{i+1}^{n+1}-\theta_{i}^{n+1}\right)^{3}-\left(\theta_{i}^{n+1}-\theta_{i-1}^{n+1}\right)^{3}\right] \\
& +\frac{\Delta t}{2 h^{2}}\left[\left(\Lambda_{i+1}^{n}+\Lambda_{i}^{n}\right)\left(\theta_{i+1}^{n+1}-\theta_{i}^{n+1}\right)-\left(\Lambda_{i}^{n}+\Lambda_{i-1}^{n}\right)\left(\theta_{i}^{n+1}-\theta_{i-1}^{n+1}\right)\right] \\
& +\frac{\Delta t}{4 h^{2}}\left(\Lambda_{i+1}^{n}-\Lambda_{i-1}^{n}\right)\left(\theta_{i+1}^{n+1}-\theta_{i-1}^{n+1}\right) \\
& -C m \frac{\Delta t}{h^{2}}\left[\sin \left(2 \theta_{i+1}^{n+1}\right)+\sin \left(2 \theta_{i-1}^{n+1}\right)-2 \sin \left(2 \theta_{i}^{n+1}\right)\right] \\
+ & C m \frac{\Delta t}{4 h^{2}}\left(\theta_{i+1}^{n+1}-\theta_{i-1}^{n+1}\right)^{2} \sin \left(2 \theta_{i}^{n+1}\right)=\theta_{i}^{n}
\end{aligned}
$$

This equation may be written in a general form as

$$
G\left(\vec{\theta}^{n+1}\right)=F
$$

and is solved by the Newton method. The equation solved at each iteration step is

$$
G\left(\overrightarrow{\theta^{s}}\right)+\frac{\partial G}{\partial \vec{\theta}}\left(\overrightarrow{\theta^{s}}\right) \Delta \vec{\theta}=F
$$

and this can be written in the form $(i=2, \ldots, N-2)$

$$
a_{i}(\Delta \theta)_{i-2}-b_{i}(\Delta \theta)_{i-1}+c_{i}(\Delta \theta)_{i}-d_{i}(\Delta \theta)_{i+1}+e_{i}(\Delta \theta)_{i+2}=f_{i} .
$$


For the coefficients $a_{i}, b_{i}, c_{i}, d_{i}, e_{i}, f_{i}$ the following expressions are valid:

$$
\begin{aligned}
a_{i}=\frac{\partial G}{\partial \theta_{i-2}}= & \frac{\Delta t}{h^{4}} \\
b_{i}=-\frac{\partial G}{\partial \theta_{i-1}}= & \frac{4 \Delta t}{h^{4}}-\frac{3 \Delta t}{2 h^{4}}\left(\theta_{i}^{s}-\theta_{i-1}^{s}\right)^{2} \\
& -\frac{\Delta t}{2 h^{2}}\left(\Lambda_{i}^{n}+\Lambda_{i-1}^{n}\right)+\frac{\Delta t}{4 h^{2}}\left(\Lambda_{i+1}^{n}-\Lambda_{i-1}^{n}\right)+C m \frac{\Delta t}{h^{2}} 2 \cos \left(2 \theta_{i-1}^{s}\right) \\
& +C m \frac{\Delta t}{2 h^{2}}\left(\theta_{i+1}^{s}-\theta_{i-1}^{s}\right) \sin \left(2 \theta_{i}^{s}\right) \\
c_{i}=\frac{\partial G}{\partial \theta_{i}}=1 & +\frac{6 \Delta t}{h^{4}}-\frac{3 \Delta t}{2 h^{4}}\left(\theta_{i+1}^{s}-\theta_{i}^{s}\right)^{2} \\
& -\frac{3 \Delta t}{2 h^{4}}\left(\theta_{i}^{s}-\theta_{i-1}^{s}\right)^{2}-\frac{\Delta t}{2 h^{2}}\left(\Lambda_{i+1}^{n}+\Lambda_{i-1}^{n}+2 \Lambda_{i}^{n}\right)+4 C m \frac{\Delta t}{h^{2}} \cos \left(2 \theta_{i}^{s}\right) \\
& +C m \frac{\Delta t}{2 h^{2}}\left(\theta_{i+1}^{s}-\theta_{i-1}^{n+1}\right)^{2} \cos \left(2 \theta_{i}^{n+1}\right) \\
d_{i}=-\frac{\partial G}{\partial \theta_{i+1}}= & \frac{4 \Delta t}{h^{4}}-\frac{3 \Delta t}{2 h^{4}}\left(\theta_{i+1}^{s}-\theta_{i}^{s}\right)^{2} \\
& -\frac{\Delta t}{2 h^{2}}\left(\Lambda_{i+1}^{n}+\Lambda_{i}^{n}\right)-\frac{\Delta t}{4 h^{2}}\left(\Lambda_{i+1}^{n}-\Lambda_{i-1}^{n}\right)+2 C m \frac{\Delta t}{h^{2}} \cos \left(2 \theta_{i+1}^{s}\right) \\
& -C m \frac{\Delta t}{2 h^{2}}\left(\theta_{i+1}^{s}-\theta_{i-1}^{s}\right) \sin \left(2 \theta_{i}^{s}\right) \\
e_{i}=\frac{\partial G}{\partial \theta_{i+2}}= & \frac{\Delta t}{h^{4}} \\
f_{i}=\theta_{i}^{n}-G\left(\theta_{i}^{s}\right) & \\
& \\
&
\end{aligned}
$$

The remaining four equations are obtained from the boundary conditions (19) and (20). Condition (19) is approximated by finite differences in the following way:

$$
C m \sin \left(2 \theta_{0}^{n+1}\right)-\frac{1}{h^{2}}\left(\theta_{2}^{n+1}+\theta_{0}^{n+1}-2 \theta_{1}^{n+1}\right)=0
$$

which for the coefficients $b_{1}, c_{1}, d_{1}, e_{1}, f_{1}$ gives

$$
\begin{aligned}
b_{1} & =-\frac{\partial G}{\partial \theta_{0}}=-2 C m \cos \left(2 \theta_{0}^{s}\right)+\frac{1}{h^{2}} \\
c_{1} & =\frac{2}{h^{2}} \\
d_{1} & =\frac{1}{h^{2}} \\
e_{1} & =0 \\
f_{1} & =\frac{1}{h^{2}}\left(\theta_{2}^{s}+\theta_{0}^{s}-2 \theta_{1}^{s}\right)-C m \sin \left(2 \theta_{0}^{s}\right) .
\end{aligned}
$$

The corresponding equation for the other end is

$$
C m \sin \left(2 \theta_{N}^{n+1}\right)-\frac{1}{h^{2}}\left(\theta_{N-2}^{n+1}+\theta_{N}^{n+1}-2 \theta_{N-1}^{n+1}\right)=0
$$

which for the coefficients $a_{N-1}, b_{N-1}, c_{N-1}, d_{N-1}, e_{N-1}, f_{N-1}$ gives

$$
\begin{aligned}
& a_{N-1}=0 \\
& b_{N-1}=\frac{1}{h^{2}}
\end{aligned}
$$




$$
\begin{aligned}
c_{N-1} & =\frac{2}{h^{2}} \\
d_{N-1} & =\frac{1}{h^{2}}-2 C m \cos \left(2 \theta_{N}^{s}\right) \\
e_{N-1} & =0 \\
f_{N-1} & =\frac{1}{h^{2}}\left(\theta_{N-2}^{s}+\theta_{N}^{s}-2 \theta_{N-1}^{s}\right)-C m \sin \left(2 \theta_{N}^{s}\right) .
\end{aligned}
$$

Finally, the condition for the absence of momentum stress at the free ends is approximated as follows:

$$
3 \theta_{0}^{n+1}-4 \theta_{1}^{n+1}+\theta_{2}^{n+1}=0
$$

which for the coefficients $c_{0}, d_{0}, e_{0}, f_{0}$ gives

$$
c_{0}=3 ; \quad d_{0}=4 ; \quad e_{0}=1 ; \quad f_{0}=4 \theta_{1}^{s}-\theta_{2}^{s}-3 \theta_{0}^{s} .
$$

For the right-hand end, the corresponding condition is

$$
-4 \theta_{N-1}^{n+1}+\theta_{N-2}^{n+1}+3 \theta_{N}^{n+1}=0
$$

which for the coefficients $a_{N}, b_{N}, c_{N}, f_{N}$ gives

$$
a_{N}=1 ; \quad b_{N}=4 ; \quad c_{N}=3 ; \quad f_{N}=4 \theta_{N-1}^{s}-\theta_{N-2}^{s}-3 \theta_{N}^{s} .
$$

The finite-difference equation for the tension is as follows $(i=2, \ldots, N-2)$ :

$$
b_{i} \Lambda_{i}^{n}-a_{i} \Lambda_{i-1}^{n}-c_{i} \Lambda_{i+1}^{n}=f_{i} .
$$

Here,

$$
\begin{aligned}
a_{i} & =1 \\
b_{i} & =2+\frac{1}{4}\left(\theta_{i+1}^{n}-\theta_{i-1}^{n}\right)^{2} \\
c_{i} & =1 \\
f_{i} & =-\frac{1}{4 h^{2}}\left(\theta_{i+1}^{n}-\theta_{i-1}^{n}\right)\left(\theta_{i+2}^{n}-2 \theta_{i+1}^{n}+2 \theta_{i-1}^{n}-\theta_{i-2}^{n}+\frac{1}{8}\left(\theta_{i+1}^{n}-\theta_{i-1}^{n}\right)^{3}\right) \\
& \quad+C m \frac{1}{2}\left(\theta_{i+1}^{n}-\theta_{i-1}^{n}\right)^{2} \cos \left(2 \theta_{i}^{n}\right)+C m \frac{1}{2}\left(( \theta _ { i + 1 } ^ { n } - \theta _ { i } ^ { n } ) \left(\sin \left(2 \theta_{i+1}^{n}\right)\right.\right. \\
& \left.\left.\quad+\sin \left(2 \theta_{i}^{n}\right)\right)-\left(\theta_{i}^{n}-\theta_{i-1}^{n}\right)\left(\sin \left(2 \theta_{i}^{n}\right)+\sin \left(2 \theta_{i-1}^{n}\right)\right)\right) .
\end{aligned}
$$

At $i=1$, equation (18) is approximated as follows:

$$
\begin{aligned}
\Lambda_{1}\left(2+\frac{1}{4}\left(\theta_{2}^{n}-\right.\right. & \left.\left.\theta_{0}^{n}\right)^{2}\right)-\Lambda_{2}-\Lambda_{0} \\
= & -\frac{1}{2 h^{2}}\left(\theta_{2}^{n}-\theta_{0}^{n}\right)\left(\theta_{3}^{n}+3 \theta_{1}^{n}-\theta_{0}^{n}-3 \theta_{2}^{n}+\frac{1}{16}\left(\theta_{2}^{n}-\theta_{0}^{n}\right)^{3}\right) \\
& +C m \frac{1}{2}\left(\theta_{2}^{n}-\theta_{0}^{n}\right)^{2} \cos \left(2 \theta_{1}^{n}\right)+C m \frac{1}{2}\left(\left(\theta_{2}^{n}-\theta_{1}^{n}\right)\left(\sin \left(2 \theta_{2}^{n}\right)+\sin \left(2 \theta_{1}^{n}\right)\right)\right. \\
& \left.-\left(\theta_{1}^{n}-\theta_{0}^{n}\right)\left(\sin \left(2 \theta_{1}^{n}\right)+\sin \left(2 \theta_{0}^{n}\right)\right)\right) .
\end{aligned}
$$

Correspondingly, at $i=N-1$,

$$
\begin{aligned}
\Lambda_{N-1}\left(2+\frac{1}{4}(\right. & \left.\left.\theta_{N}^{n}-\theta_{N-2}^{n}\right)^{2}\right)-\Lambda_{N-2}-\Lambda_{N} \\
= & -\frac{1}{2 h^{2}}\left(\theta_{N}^{n}-\theta_{N-2}^{n}\right)\left(-\theta_{N-3}^{n}-3 \theta_{N-1}^{n}+3 \theta_{N-2}^{n}+\theta_{N}^{n}+\frac{1}{16}\left(\theta_{N}^{n}-\theta_{N-2}^{n}\right)^{3}\right) \\
& +C m \frac{1}{2}\left(\theta_{N}^{n}-\theta_{N-2}^{n}\right)^{2} \cos \left(2 \theta_{N-1}^{n}\right)+C m \frac{1}{2}\left(( \theta _ { N } ^ { n } - \theta _ { N - 1 } ^ { n } ) \left(\sin \left(2 \theta_{N}^{n}\right)\right.\right. \\
& \left.\left.+\sin \left(2 \theta_{N-1}^{n}\right)\right)-\left(\theta_{N-1}^{n}-\theta_{N-2}^{n}\right)\left(\sin \left(2 \theta_{N-1}^{n}\right)+\sin \left(2 \theta_{N-2}^{n}\right)\right)\right) .
\end{aligned}
$$




\section{References}

Bossis G 2002 Proc. 8th Int. Conf. on Electrorheological Fluids and Magnetorheological Suspensions (Nice) (Singapore: World Scientific) p 884

Cebers A 2002 Phys. Rev. E 66061402

Doyle P S et al 2002 Science 2952237

Furst E M et al 1998 Langmuir 147334

Furst E M and Gast A P 1999 Phys. Rev. Lett. 824130

Gunn J M F and Warner M 1987 Phys. Rev. Lett. 58393

Landau L D and Lifshitz E M 1965 Theory of Elasticity (Moscow: Nauka) p 202

Levin Y 1999 Phys. Rev. Lett. 831159

Mayer P et al 1997 Mater. Res. Soc. Symp. Proc. 46357

Melle S et al 2002 J. Non-Newton. Mech. 102135

Sandre O et al 1999 Phys. Rev. E 591736

Sears R P 1996 Phys. Rev. Lett. 762310

Teixeira P I C et al 2000 J. Phys.: Condens. Matter 12 R4111

Tlusty T and Safran S A 2000 Science 2901328

van Roij R 1996 Phys. Rev. Lett. 763348

Warner M et al 1985 J. Phys. A: Math. Gen. 183007

Weis J J and Levesque D 1993 Phys. Rev. Lett. 712729 